Applied Mathematical Sciences, Vol. 7, 2013, no. 14, 651 - 654

\title{
The Problem of Selection of a Set of Partially Distinguishable Guards
}

\author{
Anna Gorbenko \\ Department of Intelligent Systems and Robotics \\ Ural Federal University \\ 620083 Ekaterinburg, Russia \\ gorbenko.ann@gmail.com \\ Vladimir Popov \\ Department of Intelligent Systems and Robotics \\ Ural Federal University \\ 620083 Ekaterinburg, Russia \\ Vladimir.Popov@usu.ru
}

\begin{abstract}
In this paper we consider the problem of selection of a set of partially distinguishable guards. We describe an approach to solve the problem. This approach is based on an explicit reduction from the problem to the satisfiability problem.
\end{abstract}

Keywords: partially distinguishable guards, satisfiability, NP-complete

Visual landmarks problems has been extensively studied in robotics (see e.g. [1] - [3]). In particular, the following problem was proposed in [4].

Given a polygon $P$ and a finite set of candidate guard locations $N \subset P$, can one efficiently choose the guard set $S \subseteq N$ that minimizes the number of colors required?

A point $p \in P$ is visible from point $q \in P$ if the closed segment $[p, q]$ is a subset of $P$. Let $p \leftrightarrow q$ if and only if $p$ is visible from $q$. The visibility polygon $\operatorname{Vis}(p)$ of a point $p \in P$ is defined as

$$
\operatorname{Vis}(p)=\{q \in P \mid p \leftrightarrow q\} .
$$

Since $N$ is a finite set, we can find $\operatorname{Vis}(p)$, for any $p \in N$. Also, in view of finiteness of $N$, we can consider $P$ as a finite part of two-dimensional integer 
grid. Therefore, we can consider the following decision version of the problem from [4].

ThE PROBLEM OF SELECTION OF A SET OF PARTIALLY DISTINGUISHABLE GUARDS (SG):

Instance: A grid graph $P=(V, E)$, a finite subset $N$ of the set of vertices of $P, \operatorname{Vis}(p)$, for any $p \in N$, and positive integer $k$.

QUESTION: Are there a set $S \subseteq N$ and function

$$
C: S \rightarrow\{1, \ldots, k\}
$$

such that

$$
V=\cup_{p \in S} V i s(p)
$$

and for any $p, q \in S$, if $C(p)=C(q)$, then $q \notin V i s(p)$ ?

Note that SG is NP-complete [4]. Encoding hard problems as instances of different variants of the satisfiability problem and solving them with very efficient satisfiability algorithms has caused considerable interest (see e.g. [5] [18]). We consider an explicit reduction from SG to the satisfiability problem.

Let $P=\left\{a_{1}, \ldots, a_{|P|}\right\}, N=\left\{a_{t_{1}}, \ldots, a_{t_{|N|}}\right\}$,

$$
\begin{gathered}
\varphi[1]=\wedge_{1 \leq i \leq|N|} \vee_{1 \leq j \leq k} x[i, j], \\
\varphi[2]=\wedge_{1 \leq i \leq|N|} \wedge_{1 \leq j[1]<j[2] \leq k}(\neg x[i, j[1]] \vee \neg x[i, j[2]]), \\
\varphi[3]=\wedge_{1 \leq i[1]<i[2] \leq|N|,}(\neg w[i[1]] \vee \neg w[i[2]] \vee \neg x[i, j[1]] \vee \neg x[i, j[2]]), \\
a_{t_{i[1]} \in V i s\left(a_{t_{i[2]}}\right),} \\
1 \leq j \leq k \\
\varphi[4]=\wedge_{1 \leq i \leq|V|} \vee_{1 \leq j \leq|N|} y[i, j], \\
\varphi[5]=\wedge_{1 \leq i \leq|V|} \wedge_{1 \leq j[1]<j[2] \leq|N|}(\neg y[i, j[1]] \vee \neg y[i, j[2]]), \\
\varphi[6]=\wedge_{1 \leq i \leq|V|,}(\neg y[i, j] \vee w[j]) \\
\begin{array}{c}
1 \leq j \leq|N|, \\
a_{i} \in V i s\left(a_{t_{j}}\right) \\
\xi=\wedge_{i=1}^{6} \varphi[i]
\end{array}
\end{gathered}
$$

It is easy to check that there are a set $S \subseteq N$ and function $C: S \rightarrow\{1, \ldots, k\}$ such that $V=\cup_{p \in S} V i s(p)$ and for any $p, q \in S$, if $C(p)=C(q)$, then $q \notin$ $\operatorname{Vis}(p)$ if and only if $\xi$ is satisfiable. Clearly, $\xi$ is a CNF. So, $\xi$ gives us an explicit reduction from SG to SAT. Using standard transformations (see e.g. [19]) we can obtain an explicit transformation $\xi$ into $\zeta$ such that $\xi \Leftrightarrow \zeta$ and $\zeta$ is a $3-\mathrm{CNF}$. It is easy to see that $\zeta$ gives us an explicit reduction from $\mathrm{SG}$ to 3SAT.

We have designed a generator of natural instances for the problem SG. We consider our genetic algorithms OA[1] (see [20]), OA[2] (see [21]), OA[3] (see 


\begin{tabular}{|l|lll|}
\hline time & average & $\max$ & best \\
\hline $\mathrm{OA}[1]$ & $4.2 \mathrm{~h}$ & $13.85 \mathrm{~h}$ & $17.3 \mathrm{~min}$ \\
$\mathrm{OA}[2]$ & $2.93 \mathrm{~h}$ & $11.37 \mathrm{~h}$ & $19.23 \mathrm{~min}$ \\
$\mathrm{OA}[3]$ & $3.67 \mathrm{~h}$ & $18.41 \mathrm{~h}$ & $21.41 \mathrm{~min}$ \\
$\mathrm{OA}[4]$ & $3.84 \mathrm{~h}$ & $17.2 \mathrm{~h}$ & $18.6 \mathrm{~min}$ \\
\hline
\end{tabular}

Table 1: Experimental results for SG.

[22]), and OA[4] (see [23]) for SAT. We used heterogeneous cluster. Each test was runned on a cluster of at least 100 nodes. Selected experimental results are given in Table 1.

ACKNOWLEDGEMENTS. The work was partially supported by Analytical Departmental Program "Developing the scientific potential of high school" 8.1616.2011.

\section{References}

[1] A. Gorbenko and V. Popov, The Problem of Selection of a Minimal Set of Visual Landmarks, Applied Mathematical Sciences, 6 (2012), 4729-4732.

[2] A. Gorbenko and V. Popov, A Real-World Experiments Setup for Investigations of the Problem of Visual Landmarks Selection for Mobile Robots, Applied Mathematical Sciences, 6 (2012), 4767-4771.

[3] V. Popov, Partially Distinguishable Guards, Applied Mathematical Sciences, 6587-6591.

[4] L. Erickson and S.M. LaValle, How many landmark colors are needed to avoid confusion in a polygon?, Proceedings of IEEE International Conference on Robotics and Automation, (2011), 2302-2307.

[5] A. Gorbenko and V. Popov, On the Problem of Sensor Placement, Advanced Studies in Theoretical Physics, 6 (2012), 1117-1120.

[6] A. Gorbenko and V. Popov, On the Longest Common Subsequence Problem, Applied Mathematical Sciences, 6 (2012), 5781-5787.

[7] A. Gorbenko and V. Popov, The Binary Paint Shop Problem, Applied Mathematical Sciences, 6 (2012), 4733-4735.

[8] A. Gorbenko, M. Mornev, V. Popov, and A. Sheka, The Problem of Sensor Placement, Advanced Studies in Theoretical Physics, 6 (2012), 965-967. 
[9] A. Gorbenko, V. Popov, and A. Sheka, Localization on Discrete Grid Graphs, Lecture Notes in Electrical Engineering, 107 (2012), 971-978.

[10] A. Gorbenko and V. Popov, The Longest Common Parameterized Subsequence Problem, Applied Mathematical Sciences, 6 (2012), 2851-2855.

[11] A. Gorbenko and V. Popov, Programming for Modular Reconfigurable Robots, Programming and Computer Software, 38 (2012), 13-23.

[12] A. Gorbenko and V. Popov, The set of parameterized k-covers problem, Theoretical Computer Science, 423 (2012), 19-24.

[13] A. Gorbenko and V. Popov, Clustering Algorithm in Mobile Ad Hoc Networks, Advanced Studies in Theoretical Physics, 6 (2012), 1239-1242.

[14] A. Gorbenko and V. Popov, The Problem of Finding Two Edge-Disjoint Hamiltonian Cycles, Applied Mathematical Sciences, 6 (2012), 6563-6566.

[15] A. Gorbenko and V. Popov, Footstep Planning for Humanoid Robots, Applied Mathematical Sciences, 6 (2012), 6567-6571.

[16] A. Gorbenko and V. Popov, Multiple Occurrences Shortest Common Superstring Problem, Applied Mathematical Sciences, 6 (2012), 6573-6576.

[17] A. Gorbenko and V. Popov, The Far From Most String Problem, Applied Mathematical Sciences, 6 (2012), 6719-6724.

[18] A. Gorbenko and V. Popov, Multi-agent Path Planning, Applied Mathematical Sciences, 6 (2012), 6733-6737.

[19] A. Gorbenko and V. Popov, The c-Fragment Longest Arc-Preserving Common Subsequence Problem, IAENG International Journal of Computer Science, 39 (2012), 231-238.

[20] A. Gorbenko and V. Popov, On the Problem of Placement of Visual Landmarks, Applied Mathematical Sciences, 6 (2012), 689-696.

[21] A. Gorbenko and V. Popov, Computational Experiments for the Problem of Selection of a Minimal Set of Visual Landmarks, Applied Mathematical Sciences, 6 (2012), 5775-5780.

[22] A. Gorbenko and V. Popov, Task-resource Scheduling Problem, International Journal of Automation and Computing, 9 (2012), 429-441.

[23] A. Gorbenko and V. Popov, SAT Solvers for the Problem of Sensor Placement, Advanced Studies in Theoretical Physics, 1235-1238.

\section{Received: November 1, 2012}

